\title{
IMPROVEMENT OF THE QUALITY OF TIC-CO SYSTEM PLASMA COATING BY LASER TREATMENT
}

\section{Myroslav KINDRACHUK, Aleksei SHEVCHENKO, Andrii KRYZHANOVSKYI}

National Aviation University, 1 Kosmonavta Komarova ave., 03680, Kiev, Ukraine

State Aviation Administration of Ukraine, 14 Pobedy ave., 01135, Kiev, Ukraine

E-mail:AS.avia@avia.gov.ua

Received 23 July 2015; accepted 06 June 2016

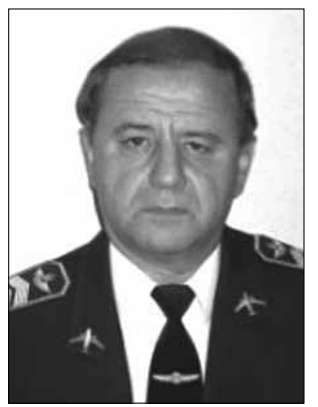

Myroslav KINDRACHUK, Prof, Dr Sci (Eng)

Education: Kiev Polytechnic Institute, 1971.

Affiliations and functions: Head of the Department of Engineering Science at the National Aviation University, Ukraine.

Research interests: tribological reliability and durability of engineering products, research and development of cobalt-base alloys for bandage blades of gas turbine engines.

Publications: author of over 250 scientific papers, including 14 monographs and manuals.

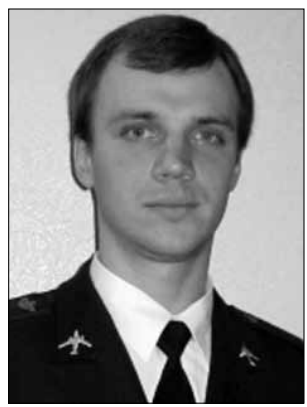

Aleksei SHEVCHENKO, MSc

Education: National Aviation University, Aerospace Institute, Aircraft Faculty, 2008.

National Aviation University, postgraduate study, 2011.

Honours, awards: Grant of the President of Ukraine, 2007.

Affiliations and functions: State Airworthiness Inspector at the Airworthiness Department at the State Aviation Administration of Ukraine, Ukraine.

Research interests: airworthiness, tribology, increasing the wear resistance of titanium alloys, composite coatings.

Publications: author of 28 scientific papers, 4 patents.

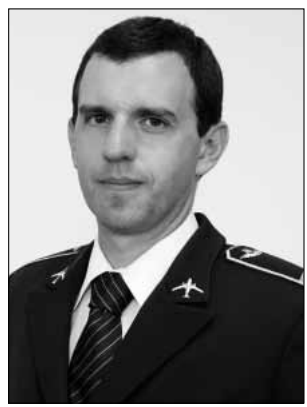

Andrii KRYZHANOVSKYI, Associate Prof, PhD (Eng)

Education: Kiev Polytechnic Institute, 1993.

Affiliations and functions: Associate Professor at the Department of Engineering Science at the National Aviation University, Ukraine.

Research interests: tribological reliability and durability of engineering products, flutter introducers.

Publications: author of over 35 scientific papers.

Abstract. The paper considers the possibility to improve the quality of a plasma coating by means of a laser treatment in the melting and thermal cycling modes. This method provides stronger adhesion of a coating to its base material as well as higher wear resistance. The regularities of the laser treatment in the melting and thermal cycling modes are determined, and a connection between the technological parameters and the quality characteristics is shown.

Keywords: wear resistance, TiC-Co plasma coating, Ti-5Al-5Mo-5V-1Fe-1Cr titanium alloy, tribotechnical characteristics, melting, thermal cycling. 


\section{Introduction}

Titanium alloys are widely used for manufacturing modern aircraft components, in particular, the pivot trunnions and inner cylinders of a nose landing gear shock-absorber, the wheel suspension lever, rack mechanism housings and steering collars of the nose landing gear steering mechanism, main landing gear breaker strut links, jack rams, etc. In order to increase the wear resistance of civil and military aircraft titanium structural elements that operate in friction units, different techniques of applying functional coatings are used. It should be noted that the technique of plasma spraying of powder materials is the most efficient and practical. This technique has been sufficiently studied and allows researchers to achieve a significant progress in extending the life time of components that operate under conditions of friction at different specific loadings and increased sliding speeds. To implement this technique, appropriate methods to select powder materials, relevant modes of applying these materials, and the necessary manufacturing equipment have been developed. The main disadvantages of the coatings obtained with the plasma method are as follows: a relatively low strength of adhesion to base materials ( $\sigma=20-30 \mathrm{MPa}$ max.) and a high porosity (6-20\%). Therefore, such coatings actually cannot be used in the case of cyclic alternating loads, especially at increased specific loads and chemically active liquids and gases (Inagaki et al. 2014; Choda et al. 2015; Liu et al. 2014; Leyens, Peters 2003).

\section{Objective of the research}

The main objective of the research is the study of a laser treatment of the TiC-Co system plasma coating in melting and thermal cycling modes. The plasma coating of the TiC-Co system is used to increase the wear resistance of aircraft equipment components made of titanium alloys.

In order to increase the mechanical and tribotechnical properties of the TiC-Co system plasma coating at Ti-5Al-5Mo-5V-1Fe-1Cr titanium alloy, the laser treatment in melting and thermal cycling modes is used.

\section{Materials and methods of research}

A complex approach and modern physical and chemical methods of analysis have been used to study the composition, structure and properties of a plasma coating and counter bodies. Their tribotechnical characteristics under conditions of friction are also determined.

The study of the tribotechnical characteristics (wear resistance and friction factor) of the initial plasma coatings applied to a Ti-5Al-5Mo-5V-1Fe-1Cr titanium alloy (Fig. 1) and the plasma coatings treated with a laser in the melting and thermal cycling modes is conducted with an advanced friction machine 2070 SMT-1. This machine

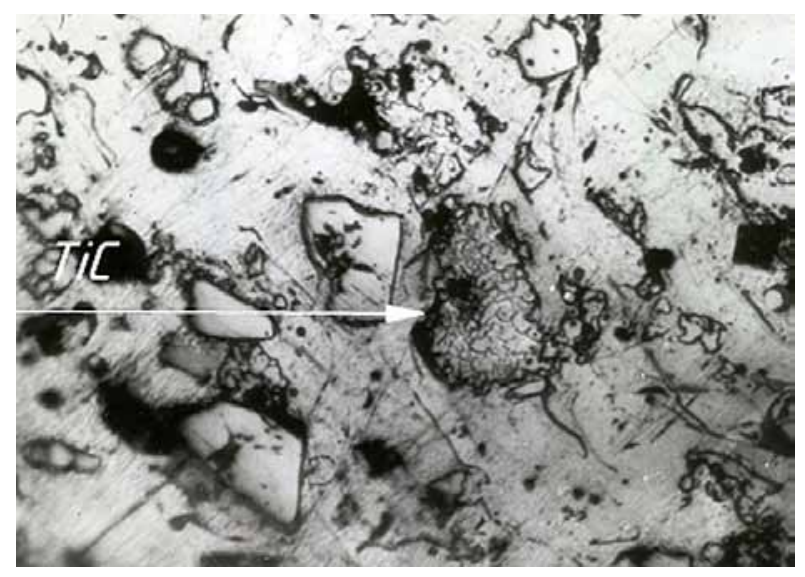

Fig. 1. Structure of the TiC-Co system plasma coating applied to a Ti-5Al-5Mo-5V-1Fe-1Cr titanium alloy, $\times 400$

allows researchers to perform the tests at sliding speeds of $v=0.1-4 \mathrm{~m} / \mathrm{s}$ (the frequency of the mating sample shaft rotation $\omega=1.25-25 \mathrm{~Hz}$ ) and loads on samples $(F=200-2000 \mathrm{~N})$, according to the model "end surface friction of a block sample and ring mating sample". Unlike the initial model of a friction machine, the advanced friction machine includes measuring equipment for the linear wear of mechanical tribosystems, as well as a computerized measuring system for obtaining and processing the tribometrical information.

Chrome coating is applied to the counter bodies of a Ti-5Al-5Mo-5V-1Fe-1Cr alloy in the form of rings with the sizes of $\varnothing 40 \times \varnothing 16 \times 10 \mathrm{~mm}$ using the galvanic method. The plasma coating is applied to Ti-5Al-5Mo$5 \mathrm{~V}-1 \mathrm{Fe}-1 \mathrm{Cr}$ titanium samples made in the form of a block. Then, they are treated to surface roughness $\mathrm{Ra} \leq$ $0.32 \mu \mathrm{m}$. The contact area of a tribocoupling does not vary under wear, which is one of the conditions of a stable process. A contact between the two elements of a friction pair is carried out under the condition of friction without lubrication. During this process, the wear rate, friction factor and temperature in the contact area are determined. The wear rate is measured using both the weight method and an inductive sensor.

\section{Results and analysis of research}

3 groups of samples of the Ti-5Al-5Mo-5V-1Fe-1Cr titanium alloy having the form of a sector are prepared. Then they are covered with a TiC-Co system plasma coating in the melting mode. The slightly etched areas are referred to as "white layers", not melted or partly melted powder particles and a small number of pore spaces are observed in the sprayed plasma coatings. The microhardness of the plasma coating matrix is $H_{0.98}=5020 \mathrm{MPa}$.

The second group of samples covered with the plasma coating is treated with a $\mathrm{CO}_{2}$ laser in the melting mode, with a beam power $P=1000 \mathrm{~W}$, the speed of a laser beam relative movement $v=0.007 \mathrm{~m} / \mathrm{s}$, and the diameter 
of the focus area of a laser beam $d=0.0025 \mathrm{~m}$. The pitch of the treatment is $S=0.0015 \mathrm{~m}$. The radiation mode provides a heating depth that is the same through the entire surface and does not exceed the thickness of the plasma coating. The ultradispersive structure is formed exactly near the surface, where the maximum rate of heat dissipation is observed. This structure is poorly detected at chemical etching. The microhardness of this structure is $H_{0.98}=4420-4595 \mathrm{MPa}$. One of the features of melted plasma coatings is a certain decrease in their microhardness in comparison with initial coatings after spraying. The main reason of such a decrease is the implementation of a mainly composite mechanism of hardening in the melted coatings in contrast to the dispersive mechanism in the initial plasma coatings. An increase in microhardness is observed all over the coating surface and the base material. Fused coatings become virtually nonporous (0.5-1.0\%); the strength of adhesion to the base material increases to $\sigma=300-350 \mathrm{MPa}$.

Tribotechnical tests have shown that the wear resistance of a melted plasma coating increases twice in comparison with the initial coating without the treatment at a temperature of $293 \mathrm{~K}$ and 5 times at a temperature of $1100 \mathrm{~K}$.

In order to develop the technological aspects of improving the characteristics of the $\mathrm{TiC}$-Co system plasma coating applied to a Ti-5Al-5Mo-5V-1Fe-1Cr alloy, several comprehensive studies are carried out. The objective of these studies is to determine the influence of laser irradiation in the thermal cycling mode which does not cause the coating fusion on the structure, adhesion to the base material, and tribological abilities of the coating (Liu et al. 2014).

Therefore, the third group of samples with an applied plasma coating is treated with $\mathrm{CO}_{2}$ laser radiation in the thermal cycling mode, at a beam power of $P=1000 \mathrm{~W}$, the speed of the laser beam relative movement of $v=0.008 \mathrm{~m} / \mathrm{s}$, and a diameter of the focus area $d=0.005-$ $0.01 \mathrm{~m}$. The pitch of the treatment is $S=0.005 \mathrm{~m}$. In this case, the laser heating varies from 1 to 6 times with the subsequent process of autocooling. This laser thermal cyclic (LTC) treatment is carried out within the temperature range of $1273 \leftrightarrow 873 \mathrm{~K}$ with a radiation power density of $40-50 \mathrm{~W} / \mathrm{mm}^{2}$ and the impact time $0.6-0.8 \mathrm{~s}$. This process includes almost the entire temperature range, which is interesting from the viewpoint of the possible phase transformations. The temperature of the upper cycle boundary is $\mathrm{T}_{\mathrm{UBC}}=0.75 \mathrm{~T}_{\text {melting }}$. At this temperature, the morphological changes in a plasma coating cannot be observed. At the same time, this temperature can cause the decomposition of the metal matrix and the coagulation of the disperse crystals that are located in the "white layers" formed during the process of spraying the plasma coating. A number of thermal cycles varies from 1 to 6 and is chosen depending on the different levels of the coating structural state which approximates to an equilibrium one (Kindrachuk et al. 2011).

The assessment of the influence of LTC treatment on the sprayed coatings' plasticity is studied on the basis of the appearance of cracks and their quantity on the prints obtained during the process of microhardness measurement using the PMT-3 and Vikkers LECO M-400 Hardness Testers. The metallographic Carl Zeiss Jena Great Tool Microscope, ORIM-1 and MIM-9 are used while studying the influence of the LTC treatment on the porosity of a plasma coating. The adhesion strength of the plasma coatings to the base material is studied on special samples using the pin method. The assessment is undertaken in order to evaluate the influence of the laser treatment in the thermal cycling mode on the value and nature of the distribution of the residual macrotensions in plasma coatings. It has been determined that the LTC treatment of the plasma coatings leads to a change in the structural state and thermodynamic balance of the "white layers". As a result, we have an increase in the quantity of areas with a more plastic structure of decomposition taking place in supersaturated solutions (Fig. 2a, b).

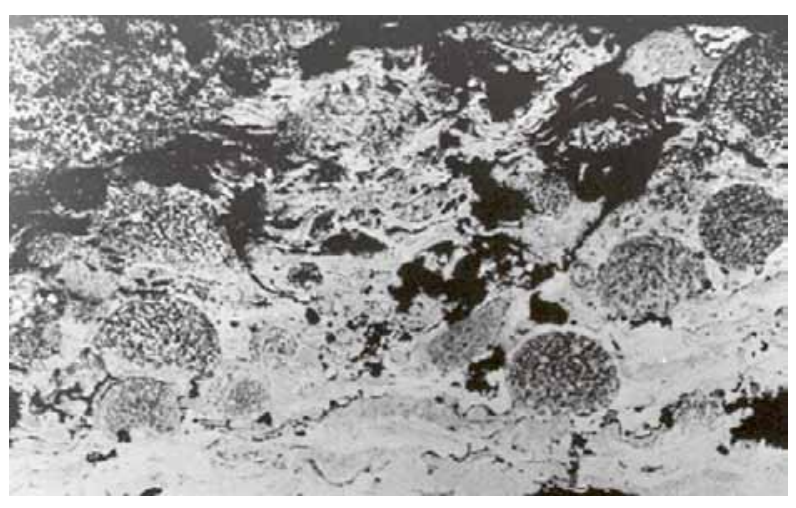

a)

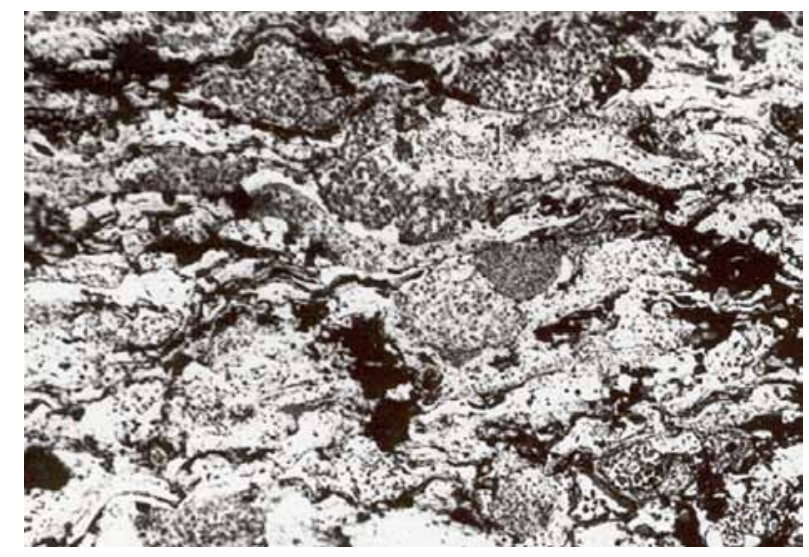

b)

Fig. 2. Initial microstructure of the plasma coating (a); and microstructure of the plasma coating after the laser treatment in the thermal cycling mode, $\times 500$ (b) 


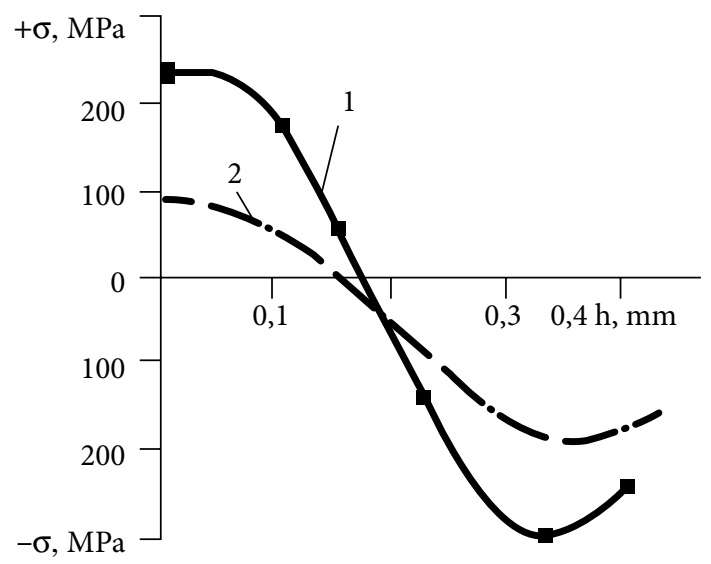

Fig. 3. Distribution of the residual tensions depending on the thickness of the TiC-Co system plasma coating on a Ti-5Al$5 \mathrm{Mo}-5 \mathrm{~V}-1 \mathrm{Fe}-1 \mathrm{Cr}$ under initial conditions (curve 1), and after 4 cycles (curve 2 )

The above mentioned structure is able to take most of the energy and reduce the tensions that occur during friction to a greater extent. Besides, the increase in the wear resistance is observed due to the increase in the modulus of the elasticity of the "white layers", which is connected with their partial disintegration. The LTC treatment stipulates a greater than twice decrease of the value of tensile residual macrotensions and a reduction of their depth gradient (Fig. 3). The level of macrotensions depends on the duration of the laser heating and the length of pauses between cycles.

It has been revealed that the LTC treatment reduces the fragility of a plasma coating as well as increases its plasticity and the strength of adhesion with the base material, and also improves the ability to construct dissipative tribological structures. The formation of metastable ultradispersive tribological structures ensures the improvement of the wear resistance of a coating at a running-in stage.

Continuous films are formed on the friction surfaces of the plasma coatings under the LTC treatment. If there is no thermal cycling on the plasma coatings, the discontinuous films will be formed as separate sectors.

It has been determined that the decomposition of the "white layers" and the significant reduction of the boundaries between them occur from the beginning of the third thermal cycle. In a TiC-Co system coating, an increase in the quantity of the "white layers" is observed after a triple treatment. These "white layers" partially decompose and turn grey (Fig. 2 b).

It has been determined that the microhardness of the matrix of the plasma coating treated by laser in the thermal cycling mode is equal to $4370 \mathrm{MPa}$ and decreases to $4010 \mathrm{MPa}$ after only the fourth thermal cycle. These processes take place owing to the fact that the metal matrix decomposes. The increase in the quantity of thermal cycles up to five leads to a practically complete decomposition of the "white layers" with the appearance of disperse particles at the interstitial phase. A continuous decrease in microhardness from 6020 to $4370 \mathrm{MPa}$, which is connected with an increase in the quantity of thermal cycles (four thermocycles), is typical for the "white layers". After six cycles, the hardness of the "white layers" and the entire plasma coating becomes approximately the same. The $\mathrm{m}$ icrohardness of a coating treated by the LTC is slightly lower than an applied coating without the LTC treatment, but is higher than that of aa melted one. A further increase in the number of irradiations leads to the reduction of the microhardness of the "white layers" due to the coagulation of interstitial phases. The LTC treatment is accompanied with the development of relaxation processes that increase the plasticity of an applied coating, which can be proved by the fact that there are no cracks in this coating and on its prints during the measurement of the microhardness.

The porosity of a coating and strength of adhesion between a coating and the base material have been investigated after a 2-6 times LTC treatment when a sufficiently high microhardness of the "white layers" and the entire coating are maintained. The research results have shown that laser thermal cycling reduces the plasma coating porosity from $7-9 \%$ to $4-6 \%$ and considerably increases the strength of adhesion between a coating and the base material from 17-20 to 80-100 MPa. Such a result is obviously associated with the processes of mass transfer from the plasma coating to the titanium alloy, and vice versa, initiated by the LTC treatment on the boundary layer between a coating and the base material with a diffusion area between them (Inagaki et al. 2014).

Friction and wear resistance tests at increased frictional temperatures show that the wear resistance of the initial plasma coating and the plasma coating after the LTC treatment are approximately identical. This can be explained by the complete decomposition of solid metastable structures and intensive oxidation of a coating because of its porosity.

Tests of titanium alloy aircraft parts to which the TiC-Co system plasma coatings are applied under manufacturing conditions have shown an increase in the wear resistance of these parts in comparison with the existing retrofitting technology.

\section{Conclusions}

1. It has been determined that the laser melting of the TiC-Co system plasma coating applied to a Ti-5Al$5 \mathrm{Mo}-5 \mathrm{~V}-1 \mathrm{Fe}-1 \mathrm{Cr}$ titanium alloy leads to the formation of pore-free structures $(0.5-1.0 \%)$ with a microhardness of 4420-4595 $\mathrm{MPa}$ and increases the strength of adhesion between a coating and the base material up to $350 \mathrm{MPa}$. However, the wear resistance slightly increases due to the complete decomposition 
of the "white layers" which foster the process of running-in and reduce the tensions generated by friction.

2. After 2-6 laser thermal cycles, the plasma coating porosity decreases from $7-9 \%$ to $4-6 \%$ and the adhesion between a coating and the base material increases up to 80-100 $\mathrm{MPa}$. Also, a sufficiently high microhardness is maintained for both the "white layers" and the entire coating. The LTC treatment creates changes in the structural conditions and thermodynamic balance of the "white layers", resulting in an increase in the number of areas with more plastic structures. These structures can absorb a significant amount of energy and reduce tensions to a greater extend during friction. A decrease in the fragility and an increase in the plasticity of a plasma coating after the LTC treatment improve its ability to form dissipative tribological structures.

3. A laser treatment of plasma coatings applied to a Ti$5 \mathrm{Al}-5 \mathrm{Mo}-5 \mathrm{~V}-1 \mathrm{Fe}-1 \mathrm{Cr}$ titanium alloy in the melting and thermal cycling modes improves the tribotechnical properties of these coatings for a wide range of temperatures. In this case, it is recommended to use sprayed coatings with an additional LTC treatment for the parts of friction units of aircraft equipment that operate under low-temperature conditions. For high temperature conditions, melted plasma coatings should be applied.

\section{References}

Choda, T.; Oyama, H.; Murakami, S. 2015. Technologies for process design of titanium alloy forging for aircraft parts, Kobelco Technology Review 33: 44-49.

Inagaki, I.; Takechi, T.; Shirai, Y., et al. 2014. Application and features of titanium for the aerospace industry, Nippon Steel \& Sumitomo Metal Technical Report 106: 22-27.

Kindrachuk, M.; Shevchenko, A.; Dukhota, O., et al. 2011. A method to form wearproof gas-thermal coatings by laser thermal cycling. Patent No 65209. Kiev: Applicant and Patentee National Aviation University.

Liu, S.; Liu, Z.; Wang, Y., et al. 2014. Ti-based composite coatings with gradient $\mathrm{TiCx}$ reinforcements on TC4 titanium alloy prepared by laser cladding, Science China Technological Sciences 57: 1454-1461. http://dx.doi.org/10.1007/ s11431-014-5566-5

Leyens, C.; Peters, M. 2003. Titanium and titanium alloys. Fundamentals and applications. Wiley-VCH Verlag $\mathrm{GmbH} \&$ Co. KGaA. 513 p. http://dx.doi.org/10.1002/3527602119 